\title{
Deposition of zinc films by laser method
}

\author{
V.K. Goncharov, G.A. Gusakov, M.V. Puzyrev
}

\begin{abstract}
Conditions of laser irradiation of a zinc target under which large droplets of a laser target material are not formed in the erosion plume are found, and zinc nanofilms with a minimum number of large particles on the surface are produced. The surface structure, thickness and optical characteristics of zinc films are determined as functions of the power density of laser radiation falling on a zinc target. The evaporation threshold for a zinc target irradiated by nanosecond laser pulses is found.
\end{abstract}

Keywords: laser-plasma deposition, nanosized zinc films, laser erosion plasma.

\section{Introduction}

Transparent flexible conductors are important components of modern electronic and optoelectronic devices such as interactive electronics, solar energy, implantable medical devices and various robotic systems. Thus, the problem of producing uniform nanoscale coatings at low temperatures, which do not exceed the softening temperature of the substrate material, and without subsequent annealing is very urgent, especially in the deposition of coatings on polymeric substrates.

One of the most promising methods for the preparation of nanostructured films is the pulsed laser deposition technique [1], which consists in sputtering a target in a vacuum chamber by laser pulses and in depositing a target material on a substrate. The advantages of this method are its simplicity and equally good evaporation of all chemical elements contained in a target [2]. By evaporating a target under certain conditions it is possible to obtain a film with a desired stoichiometric composition.

In many important technological processes the deposited metal coatings should be uniform and have a minimum number of frozen metal droplets on the film surface.

It is known that the formation of a liquid-phase phase in laser erosion plumes of metals may be caused by three mechanisms [3-7].

The largest liquid droplets are formed due to the hydrodynamic mechanism [3-5]. Their size depends on many factors such as the spatiotemporal shape of laser pulses, focusing conditions, purity of a target material and type of metal. The diameter of these particles is $1-100 \mu \mathrm{m}$.

V.K. Goncharov, G.A. Gusakov, M.V. Puzyrev A.N. Sevchenko Institute of Applied Physical Problems of Belarusian State University, ul. Kurchatova 7, 220045 Minsk, Belarus; e-mail: puzyrev@bsu.by

Received 11 December 2013; revision received 13 June 2014

Kvantovaya Elektronika 45 (4) 339-344 (2015)

Translated by I.A. Ulitkin
Volume vaporisation processes also result in the formation of the target material particles (depending on the laser irradiation conditions) with a diameter of $0.04-1 \mu \mathrm{m}$ [6].

When metal targets are irradiated by single laser pulses of short duration, target material particles are mainly formed in the laser erosion plume due to condensation of degradation products [7]. Their diameter is typically $40-60 \mathrm{~nm}$.

In the presence of synergistic processes, when laser pulses irradiate metal targets in the frequency regime, particles of the condensed phase of the target material may be formed in a laser erosion plume at the expense of all three mechanisms. In this case, the predominance of one or another mechanism is dependent on the irradiation conditions (laser energy in a single pulse, focusing conditions, laser pulse repetition rate, rate of movement of the laser beam along the target surface, etc.).

Currently, there are several ways of forming laser erosion plumes with a minimum number of large droplets of particles of the laser target material.

One of the easiest is to place a screen in the area of the main flow of the laser target degradation products [8]. However, this method involves the use of the particles at the periphery of the laser erosion plume and the presence of a buffer gas in a vacuum chamber. As a result, the film is deposited with slow particles whose velocity is comparable with that of the particles in thermal sputtering. The coating deposition velocity is significantly reduced, and in some cases the required adhesion of the coating to the substrate material is not achieved.

In [9], based on the study of the dynamics of the laser erosion plume, a method is proposed to eliminate large droplets by placing a diaphragm, which holds large droplets and passes the plasma plume [10]. In this case, the main laser target degradation products effectively participate in the deposition of films. Burimov et al. [11] proposed a method of removing large, low-velocity droplets by placing a rotating disk with an aperture in the way of the erosion plume. In this case, the disk rotation and the high-velocity component of the erosion plume should be synchronised so that the aperture was opposite the substrate at the moment of the laser pulse arrival. Thus, the droplets are prevented from reaching the film surface and are trapped on the disk. Gorbunov et al. [12] proposed cross-beam pulsed laser deposition when two laser beams are directed to two targets at different angles and erosion plumes intersect at a certain angle. As a result of elastic and inelastic collisions of the fastest particles, a new plasma plume, free of large droplets, is formed.

A more effective method of obtaining a laser erosion plume without large droplets was proposed in [13]. It is based on the formation of a laser erosion plume by the first laser beam and on additional evaporation of liquid droplets by the 
second laser beam propagating perpendicularly to the erosion plume.

Of the following methods of removal of large droplets, most effective are the last three methods. However, they have some drawbacks. One should take into account that the method of removing particles with the help of a rotating disk having an aperture involves synchronisation of the laser plume appearance with the appearance of the aperture in the rotating disk. Because the rotation of the disk cannot be fundamentally synchronised, the reference synchronisation pulse should be generated by the disk rotation system itself, which is particularly difficult to realise. Furthermore, compared to other electronic systems this system is less stable. To implement the last two methods requires the use of two lasers, which imposes additional conditions (purchase of fairly sophisticated and expensive equipment) on the deposition technology. The system of their synchronisation is also complex. It should be noted that the radiation beam from a single laser can be split into two and each of the beams can be delivered to the desired location using a complicated optical system. However, this requires the use of a high-energy (and therefore more expensive) laser. Thus, the separation of radiation into two beams does not greatly simplify the task.

Thus, the objective of this paper is to find such regimes of laser irradiation of metals, when a liquid-metal layer is not formed in the exposed region and large droplets of the target material are not produced in the erosion plume due to the hydrodynamic mechanism, as well as to develop a simple method of laser-plasma film deposition using a single laser without any additional optical systems and rigid synchronisation of mechanical and optical systems. This will significantly simplify the production technology of nanoscale films and will make it cheaper.

\section{Experiment}

For film deposition we used a 1064-nm pulsed LS-2137 YAG: $\mathrm{Nd}^{3+}$ laser (Lotis-TII) with a FWHM pulse duration $\tau=20 \mathrm{~ns}$. The laser pulse repetition rate was $10 \mathrm{~Hz}$, and the deposition continued for $1 \mathrm{~h}$. The target made of commercially pure zinc was placed at an angle of $45^{\circ}$ to the axis of the laser beam. As a substrate we used silicon and glass. The deposition was performed in vacuum at a residual gas pressure of $\sim 10^{-3} \mathrm{~Pa}$ or in the air atmosphere. The substrates were placed parallel to the target surface, and the distance $L$ between the substrate and the target was 25 or $100 \mathrm{~mm}$. The target was rotated at $2 \mathrm{rpm}$, to prevent the formation of deep craters on its surface, which may affect the spatial shape of the erosion plume. The erosion trace on the target was a circle of varying width and diameter of 15-20 $\mathrm{mm}$ (depending on the type of the target and the conditions of the experiment).

The surface structure of the films was studied using LEO 1455 VP (Carl Zeiss, Germany) and S-4800 (Hitachi, Japan) scanning electron microscopes. The coating thickness was determined with a profilometer (Talystep, USA). Spectral characteristics of the deposited film in the range 200-900 nm were studied using a Cary 300 spectrophotometer (Varian, USA). The parameters of the erosion crater on the surface of the target were measured with an MKTF-1 optical microscope.

\section{Results and discussion}

For a metal to be efficiently deposited, it is important to know the threshold laser power density, at which the target material starts to intensively evaporate. Since the energy distribution over the laser beam cross section is close to Gaussian, we should determine some averaged laser power density in the destruction of the target. As an average power density of laser radiation, we used the ratio of the laser pulse energy to its duration and the area of the laser spot on the target surface. Note that in this case the maximum power density with allowance for the Gaussian energy distribution in the spot exceeds the average power density by approximately three times. In our experiments, the average power density of the laser pulse, $P_{\mathrm{d}}$, was varied in the range from $9.9 \times 10^{7}$ to $8.9 \times$ $10^{8} \mathrm{~W} \mathrm{~cm}^{-2}$. Thus, taking into account the Gaussian energy distribution in the laser spot the maximum power density $P_{\mathrm{D}}$ ranged from $3 \times 10^{8}$ to $2.7 \times 10^{9} \mathrm{~W} \mathrm{~cm}^{-2}$.

To assess the threshold laser power density, we measured the linear dimensions of the erosion craters produced on the target surface as a result of exposure to laser radiation with different power densities. The experiments showed that at a laser pulse energy of $140 \mathrm{~mJ}$ and a beam spot diameter $d_{\mathrm{s}} \sim 1 \mathrm{~mm}$, the crater diameter was $1.25 \mathrm{~mm}$, at $d_{\mathrm{s}}=2 \mathrm{~mm}-$ $1.4 \mathrm{~mm}$ and at $d_{\mathrm{s}}=3 \mathrm{~mm}-0.4 \mathrm{~mm}$, which is associated with a decrease in $P_{\mathrm{D}}$ with increasing diameter of the laser spot. At a laser beam diameter of $3 \mathrm{~mm}$ the power density at the spot edges is insufficient to start efficient evaporation of the target material. With decreasing diameter of the laser beam to $1 \mathrm{~mm}$ the local power density of laser radiation is increased by approximately 10 times, and it is already sufficient to start the evaporation of the target material even at the peripheral part of the laser spot. In addition, at a higher power density, the laser pulse due to the thermal conductivity and a significant temperature gradient heats a region at a considerable distance from the irradiated area. As a result, the erosion crater diameter is greater than the diameter of the laser spot. Based on the above described data and taking into account the Gaussian energy distribution in the spot (Fig. 1), we can make a conclusion that for zinc the threshold power density $P_{\mathrm{D}}^{\text {th }}=2.7 \times$ $10^{8} \mathrm{~W} \mathrm{~cm}^{-2}$, which corresponds to $P_{\mathrm{d}}=0.9 \times 10^{8} \mathrm{~W} \mathrm{~cm}^{-2}$.

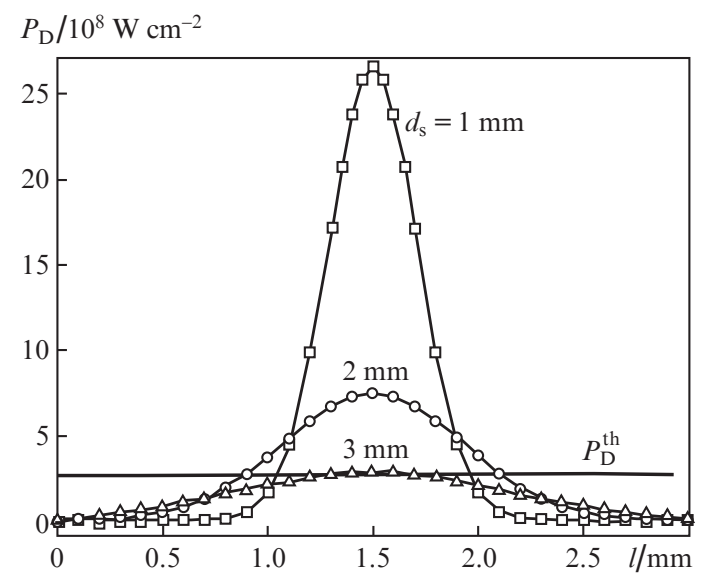

Figure 1. Laser power density distribution along the diameter of the beam spot at different values of $d_{\mathrm{s}}$

Nanoparticles of the liquid-phase target material, formed in the erosion plume due to the volume evaporation and condensation, are carried away by ions and atoms of the target material. When falling onto the substrate surface, they form nanofilms. 
Particles formed in the erosion plume by a hydrodynamic mechanism have a sufficiently large diameter $(d=1-15 \mu \mathrm{m})$ and much lower velocities compared to the velocities of atoms and ions. These particles, reaching the substrate surface, produce an uneven relief on the deposited film.

In this connection, it is necessary to determine the regimes of deposition of metal films at which their surface will contain a minimum amount of large particles. Figure 2 shows the photographs of zinc films, obtained using an electron microscope under different deposition conditions. Figure 3 presents the total number of particles on the film surface as a function of the average power density of laser radiation. From the results it follows that the number of particles on the film surface decreases with decreasing average power density of laser radiation, and at low values of $P_{\mathrm{d}}$ near the evaporation threshold of zinc one can obtain practically drop-free films.

Figure 4 shows particle-size distributions $(0.1-5 \mu \mathrm{m})$ under various experimental conditions. It is seen that with increasing size the number of particles decreases. In reality, one can see large particle on the photographs, but they are
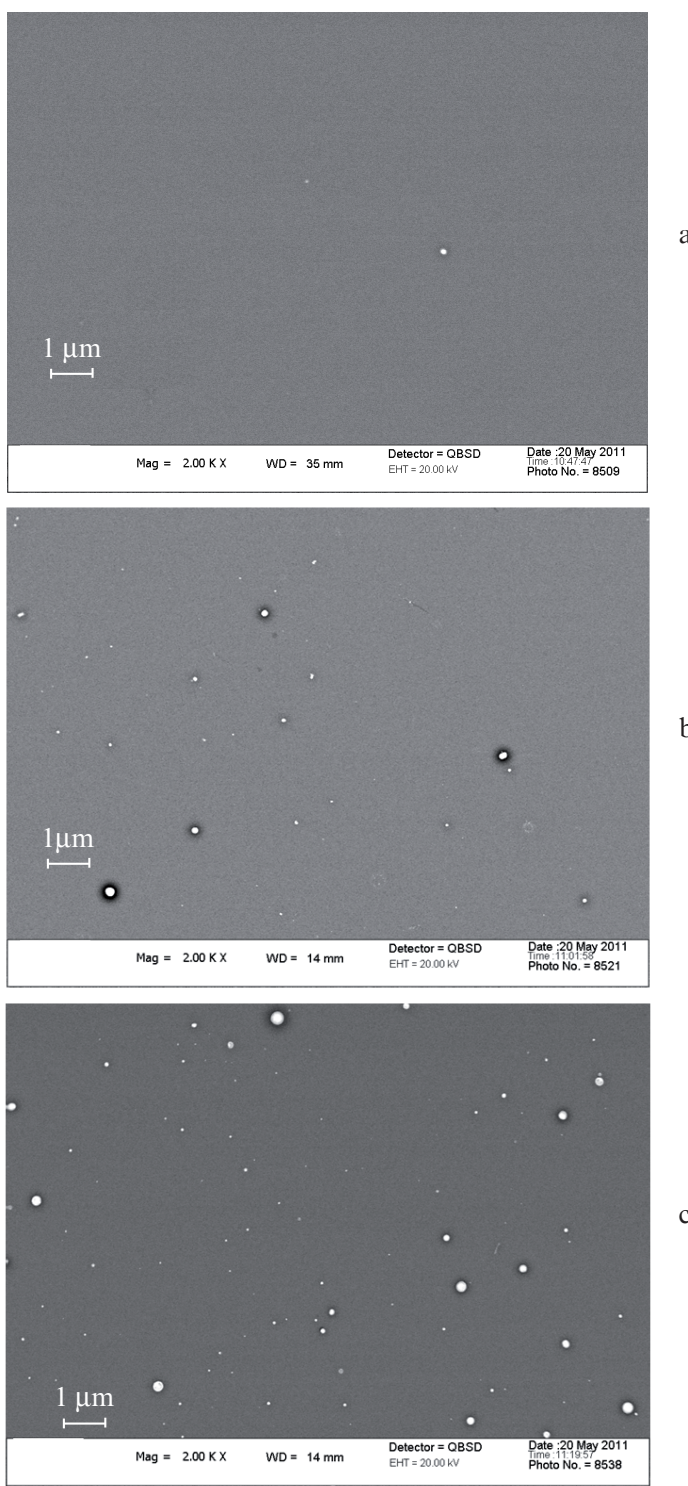

Figure 2. Surface of a zinc film at $P_{\mathrm{d}}=$ (a) $0.99 \times 10^{8}$, (b) $2.2 \times 10^{8}$ and (c) $8.9 \times 10^{8} \mathrm{~W} \mathrm{~cm}^{-2}$.

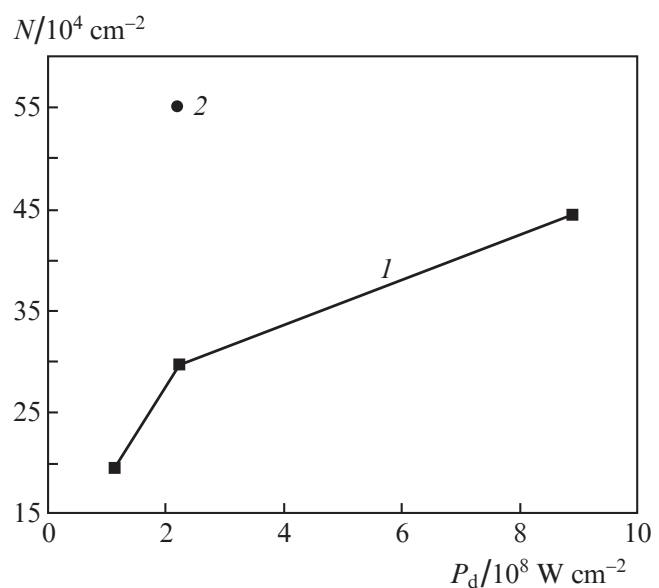

Figure 3. Dependences of the number $N$ of particles per $1 \mathrm{~cm}^{2}$ on $P_{\mathrm{d}}$ at $L=$ (1) 100 and (2) $25 \mathrm{~mm}$.

quite rare. The number of particles, whose size is less than $0.1 \mu \mathrm{m}$, is much greater. These particles increase the growth rate of the deposited coating and do not affect the uniformity of the deposited coating surface. It is also evident that with decreasing $P_{\mathrm{d}}$, the number of particles per $1 \mathrm{~cm}^{2}(N)$ on the film surface decreases.

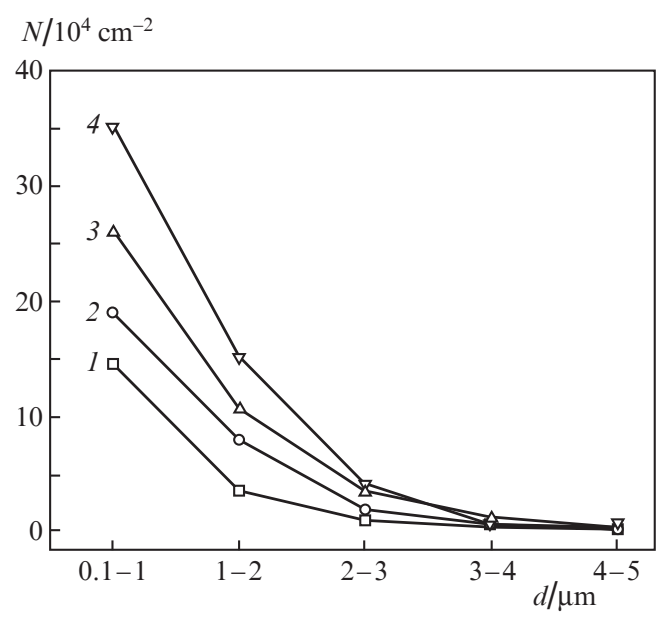

Figure 4. Dependences of the particle-size density distribution, $d$, on $P_{\mathrm{d}}=$ (1) $1.1 \times 10^{8}$, (2) $2.2 \times 10^{8}$ and (3) $8.9 \times 10^{8} \mathrm{~W} \mathrm{~cm}^{-2}$ at $L=$ $100 \mathrm{~mm}$ and on $P_{\mathrm{d}}=$ (4) $2.2 \times 10^{8} \mathrm{~W} \mathrm{~cm}^{-2}$ at $L=25 \mathrm{~mm}$.

The experiments [curves $(1-3)]$ were performed at a distance $L=100 \mathrm{~mm}$ between the substrate and the laser target. To test the effect of the distance from the substrate to the target on the number of particles deposited on the film surface, we performed an additional experiment, in which the substrate was placed at distance of $25 \mathrm{~mm}$ from a zinc target. Figure 4 [curve (4)] shows that when the distance between the substrate and the target decreases, the number of large particles increases.

This may be due to the fact that the large particles formed due to the hydrodynamic mechanism fly apart in the erosion plume at smaller angles to the surface of the zinc target than other degradation products [9]. With increasing distance from the target surface to the substrate, the concentration of large particles in the plume is reduced, and consequently, the num- 
ber of particles on the substrate surface decreases. However, the deposition rate of metal films decreases in this case.

Since the deposition rate of the film varies as a function of the laser power density, we measured the thicknesses of the films deposited under different experimental conditions. The results obtained are presented in Fig. 5. The experiments show that the dependence of the thickness of the deposited films on the laser power density is close to linear.

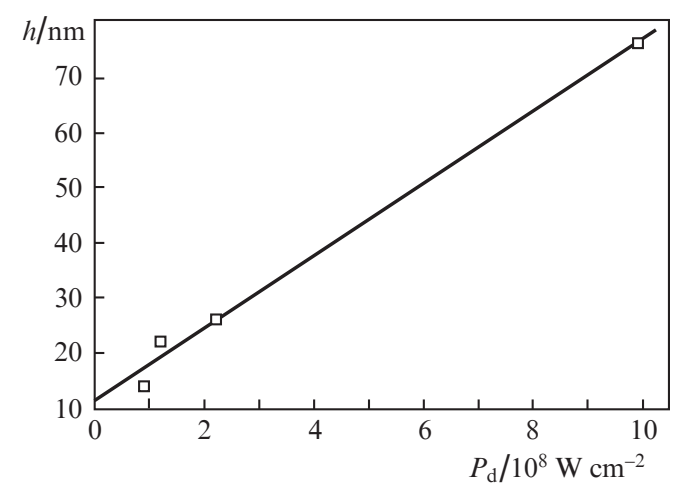

Figure 5. Dependence of the thickness $h$ of deposited films on $P_{\mathrm{d}}$ for a zinc target.

To study the influence of the laser radiation parameters on the energy characteristics of zinc plasma, we performed time-of-flight probe measurements, as described in [14]. As an ion probe use was made of a $20 \times 20-\mathrm{mm}$ copper plate. The distance between the target and the collector in all experiments was $10 \mathrm{~cm}$. The signal from the probe was detected by a Tektronix TDS 2022B speed oscilloscope.

Figure 6 shows the time dependences of the probe ion current. The zinc target was irradiated by laser pulses of different average power density. It should be noted that at a low $\left[(1-1.5) \times 10^{8} \mathrm{~W} \mathrm{~cm}^{-2}\right]$ average laser power density, the ion current through the probe is not detected. It follows that in the vicinity of the threshold values of $P_{\mathrm{d}}$, the surface of the zinc target evaporates mostly neutral particles. In the range of $P_{\mathrm{d}}=(1.8-10) \times 10^{8} \mathrm{~W} \mathrm{~cm}^{-2}$, the probe ion current is clearly measured, and for the maximum power densities, studied in our experiment, the plasma plume contains a pro-

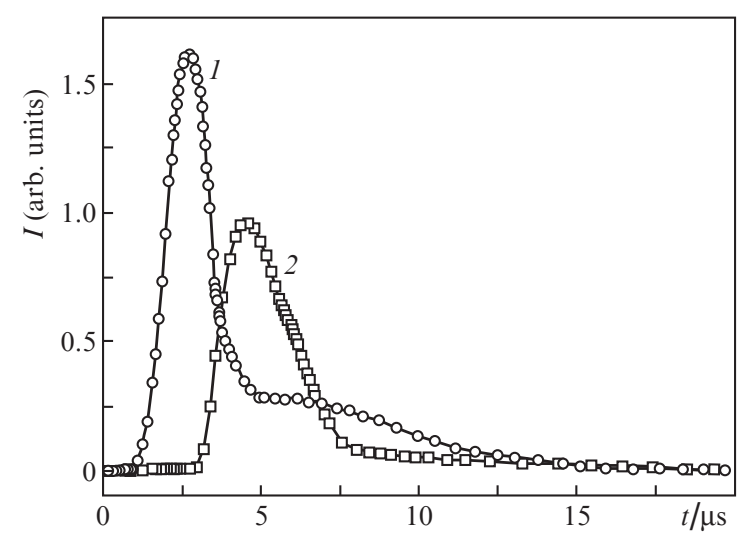

Figure 6. Time dependences of the probe ion current of the erosion plasma at $P_{\mathrm{d}}=(1) 8.9 \times 10^{8}$ and (2) $2.2 \times 10^{8} \mathrm{~W} \mathrm{~cm}{ }^{-2}$. nounced front of fast ions, followed by a 'tail' of slow particles. This is consistent with the results of Navodvorskii [15], who considered the time-of-flight characteristics of ions in plasmas of many metals, including zinc. A similar pattern was observed in [14] for the case of laser erosion of graphite. Bulgakova et al. [14] attributed this effect to the acceleration of the particles as a result of the absorption of part of the laser pulse energy by a plasma plume. Thus, it can be assumed that ionisation of the laser erosion products of a zinc target occurs predominantly due to absorption of part of the laser energy by a vapour-gas cloud formed over the target surface. At a low laser power density, the zinc vapour density above the target surface is small, laser radiation absorption in the vapour-gas cloud is insignificant and, as a result, the degree of the erosion plume ionisation is small.

Using the results of time-of-flight measurements shown in Fig. 6, we also estimated the average energy of ions in the zinc plasma for different values of $P_{\mathrm{d}}$. The average ion energy was calculated by the expression

$$
E_{\mathrm{kin}}=\frac{1}{2} \frac{\int_{0}^{\infty} m[u(t)]^{2} I(t) \mathrm{d} t}{\int_{0}^{\infty} I(t) \mathrm{d} t}=\frac{m L^{2}}{2} \frac{\int_{0}^{\infty} t^{-2} I(t) \mathrm{d} t}{\int_{0}^{\infty} I(t) \mathrm{d} t},
$$

where $I(t)$ is the ion current through the probe; $m$ is the mass of the ions; and $u(t)=L / t$ is the ion velocity.

It is shown that at $P_{\mathrm{d}}=(2.2-10) \times 10^{8} \mathrm{~W} \mathrm{~cm}^{-2}$, the kinetic energy of the particles in the erosion plume increases from 134 to $360 \mathrm{eV}$ with increasing laser power density. It follows from these estimates that due to the low melting point and low sublimation energy of zinc, this ion energy is sufficient for activation of various ion-stimulated processes on the surface of the deposited film. However, the study of the influence of the energy characteristics of the zinc plasma on the structure and characteristics of the deposited films should be more detailed and requires a separate study.

Since the deposited films are visually transparent, we measured their spectral characteristics. Figure 7 shows at different scales the spectral characteristics of the loss factor (extinction) under different deposition conditions. One can see that the maximum loss is observed in films deposited at higher power densities of incident laser radiation, which may be attributed to the film thickness and the presence of larger particles of the target material on the film surface.

At a low power density, a thin droplet-free zinc film is formed on the substrate surface and the radiation loss due to low absorption and scattering is minimal [Fig. 7, curve (1)]. When the power density of incident laser radiation is increased, small droplets appear on the surface of the zinc film. The loss factor of such films is determined not only by their greater thickness, but also by scattering by deposited droplets. According to the Love-Mie theory the main radiation losses in this case are determined by the scattering whose intensity is $1 / \lambda^{4}$, where $\lambda$ is the radiation wavelength. Therefore, with increasing power density of incident laser radiation the transmission of the deposited films decreases, especially in the short-wavelength region of the spectrum [Fig. 7, curves $(2,3,4)]$.

Figure 8 a shows the spectral characteristics of zinc films deposited at different distances between the laser target and the substrate. With decreasing distance, more liquid droplets formed in the laser erosion plume due to the hydrodynamic mechanism fall onto the substrate, i.e., such droplets move at 

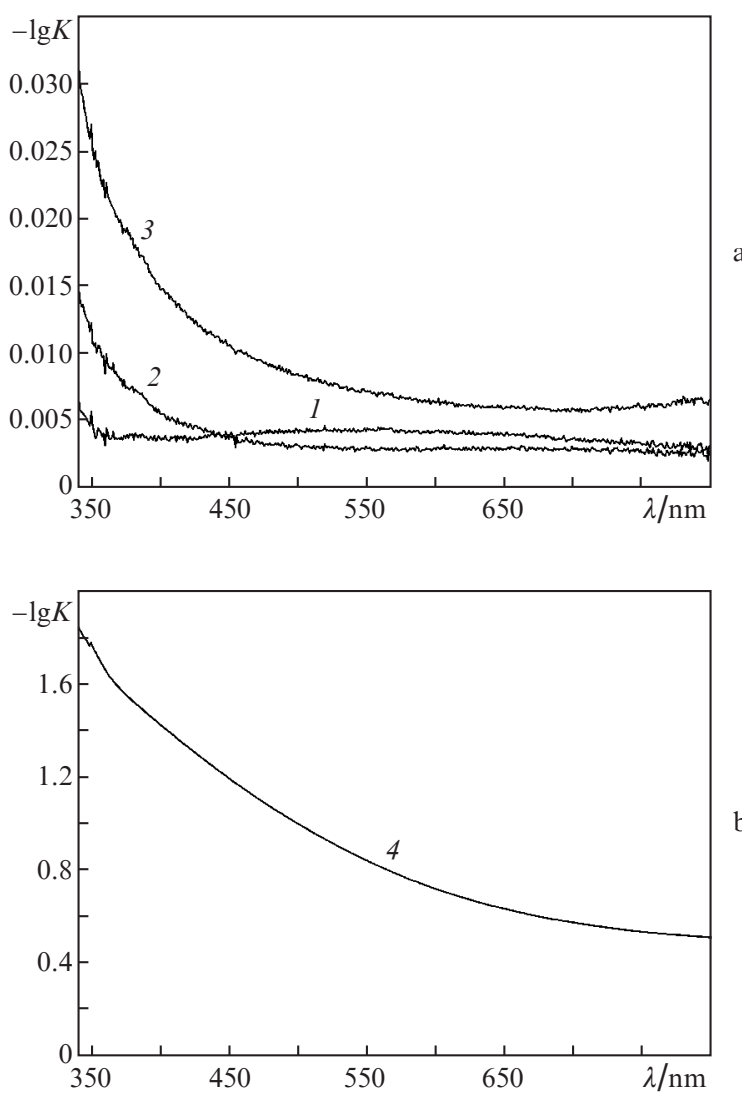

Figure 7. Spectral characteristics of the relative loss factor $K$ of deposited films at $P_{\mathrm{d}}=$ (1) $0.99 \times 10^{8}$, (2) $1.2 \times 10^{8}$, (3) $2.2 \times 10^{8}$ and (4) $8.9 \times 10^{8} \mathrm{~W} \mathrm{~cm}^{-2}$.

small angles to the target surface [9]. In this case, the droplet size exceeds the wavelength of light, and the light loss is determined by the complex refractive index of a bulky zinc target rather than by the dependence $1 / \lambda^{4}$. Therefore, in the shortwavelength region of the spectrum the loss factor of the film varies more smoothly, and its total growth in comparison with a film deposited at $L=100 \mathrm{~mm}$ is determined by an increase in the number of large droplets on the film surface and its greater thickness, because in this case the laser erosive plume scatters less and the density of erosion products in it is higher.

As a result, films deposited at shorter distances $L$ have a higher optical density, and their quality is worse due to the introduction of liquid droplets.

In experiments under low vacuum we observed a significant increase in the loss factor of the deposited zinc films in the short-wavelength region. To confirm this, we investigated the deposition of a zinc film at different distances between the target and the substrate in air at atmospheric pressure (Fig. 8b). We found that in this case, apart from a zinc film, a $\mathrm{ZnO}$ film is intensively deposited on the substrate. For the film deposited at $L=25 \mathrm{~mm}$, the loss spectra exhibit the $\mathrm{ZnO}$ absorption edge at a wavelength of $337 \mathrm{~nm}$ (Fig. 8b). This can be seen in the shortwavelength region of the spectrum of the deposited film. When removing the substrate from the target, the film is hardly deposited because the products of the laser erosion of the target material at atmospheric pressure do not reach the substrate.
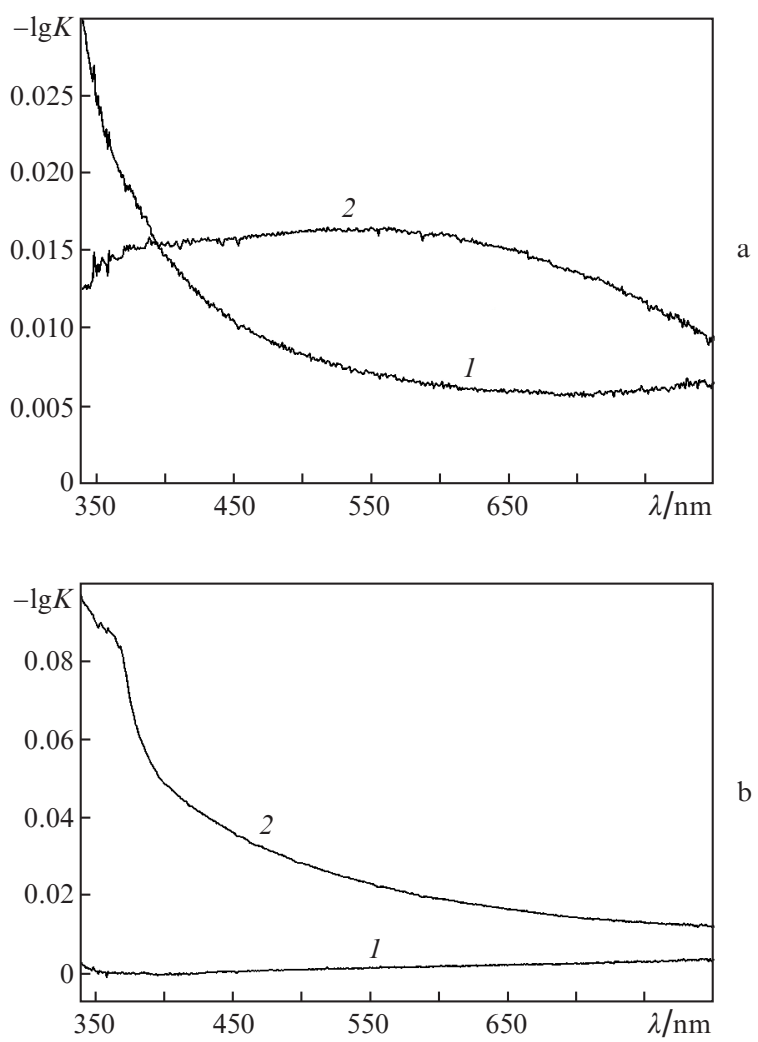

Figure 8. Spectral characteristics of the relative loss factor $K$ of deposited films upon irradiation of a zinc target by laser radiation with a power density of $2.2 \times 10^{8} \mathrm{~W} \mathrm{~cm}^{-2}$ at $L=$ (1) 100 and (2) $25 \mathrm{~mm}$ in (a) vacuum and (b) air.

\section{Conclusions}

We have found conditions of laser irradiation of a zinc target, under which large droplets of the target material in the erosion plumes are not formed, and have proposed a simple method for the deposition of zinc nanofilms on a substrate with a minimum number of large particles on its surface.

We have determined the threshold laser power density, at which zinc starts to evaporate intensely. According to our data, it is $2.7 \times 10^{8} \mathrm{~W} \mathrm{~cm}^{-2}$.

We have shown that the thickness of deposited films in our case has a linear dependence on the power density of incident laser radiation.

We have demonstrated the principal possibility of synthesis of $\mathrm{ZnO}$ films by the proposed method.

\section{References}

1. Konoplev B.G., Ageev O.A. Izv. YuFU. Tekhn. Nauki, 89 (12), 165 (2008).

2. Caricato A.P., Creti A., Luchesa A., Lomascolo M., Martino M., Rella R., Valerini D. Laser Phys., 21 (3), 588 (2011).

3. Ulyakov P.I. Zh. Eksp. Teor. Fiz., 52 (3), 820 (1967).

4. Putrenko O.I., Yankovskii A.A. Zh. Prikl. Spektrosk., 15 (4), 596 (1971).

5. Anisimov S.I., Imas Ya.A., Romanov G.S., Khodyko Yu.V. Deistvie lazernogo izlucheniya bol'shoi moshchnosti na metally (Interaction of High-Power Laser radiation with Metals) (Moscow: Nauka, 1970).

6. Goncharov V.K. Inzh.-Fiz. Zh., 74 (5), 87 (2001).

7. Goncharov V.K., Kozadaev K.V. Inzh.-Fiz. Zh., 83 (1), 1 (2010). 
8. Kinoshita K., Ishibashi H., Kobayashi T. Jpn. J. Appl. Phys., 33, 417 (1994).

9. Goncharov V.K., Kozadaev K.V., Puzyrev M.V., Slavashevich D.L. Inzh.-Fiz. Zh., 81 (2), 211 (2008).

10. Goncharov V.K., Kozadaev K.V., Puzyrev M.V. Lazernyi metod polucheniya suspenzii nanochastits metallov s pomoshch'yu prostranstvennogo separirovaniya (Laser Method of Producing Metal Nanoparticle Suspensions Using Spatial Separation). Patent for the Invention of the Republic of Belarus No. 10974 dated 05.07.2008.

11. Burimov V.N., Zherikhin A.N., Popkov V.L. Kvantovaya Elektron., 23 (1), 73 (1996) [Quantum Electron., 26 (1), 71 (1996)].

12. Gorbunov A., Tselev A., Pompe W. Proc. SPIE Int. Soc. Opt. Eng., 3688, 351 (1999).

13. Goncharov V.K., Puzyrev M.V. Kvantovaya Elektron., 24 (4), 329 (1997) [Quantum Electron., 27 (4), 319 (1997)].

14. Bulgakova N.M., Bulgakov A.V., Bobrenok O.F. Phys. Rev. E, 62 (4), 5624 (2000).

15. Navodvorskii O.A. Doct. Diss. (Shatura, IPLIT 2012). 